\title{
Under Nutrition and its Association With Infant and Young Child Feeding Summary Index Among 6 to 23 Months In Demba Gofa Woreda, Southern Ethiopia
}

\author{
Aygota Oyda ${ }^{1}$, Dessalegn Tamiru' ${ }^{2}$, Amanuel Tesfay ${ }^{2}$, Niguse Mekonnen ${ }^{3^{*}}$ \\ ${ }^{1}$ Child health and Nutrition officer, Gammo Gofa zone, Health Department, Arbaminch \\ ${ }^{2}$ Assistant professor, Department of population and Family health, College of Health Sciences, Jimma University, Jimma, Ethiopia \\ ${ }^{3}$ Lecturer, School of Public Health, College of Health Sciences and Medicine, Wolaita Sodo University, Wolaita Sodo, Ethiopia
}

Received: February 25, 2017; Accepted: April 12, 2017; Published: May 08, 2017

*Corresponding author: Niguse Mekonnen, Lecturer, School of Public Health, College of Health Sciences and Medicine, Wolaita Sodo University, Wolaita Sodo, Ethiopia, Tel: +251916704274; E-mail: nigusemekonnen69@gmail.com

\begin{abstract}
Background: Infant and Young child feeding practices remain a common problem in Ethiopia. Infant and young child practices are multidimensional and changes rapidly. The previously developed indicators could not be able to show the simultaneous effect of different dimension of complementary feeding and has focused on single practices over a narrow age range and has not addressed the impact of adequate or optimal infant and child feeding. Thus this study had shown the simultaneous effect of feeding practice in the study area in particular and in Ethiopia at large via summary index.
\end{abstract}

Objective: To assess nutritional status and its association with infant and young child feeding summary index among 6-23 months age children in Demba Gofa Woreda, SNNPR, Ethiopia.

Method: A community-based cross-sectional study design with multistage simple random sampling technique was conducted from March to May 2016 in Demba Gofa Woreda, Southern Ethiopia. The data were collected from 696 randomly selected child-mother pairs. Chi-square tests for trends and binary logistic regression for bivariate analysis and multiple logistic regressions were used for multivariable analysis to identify the association between nutritional status and infant and child feeding index terciles.

Results: The analyses revealed that 43.8\% (95\% CI: 40.1-47.5), 15.8\% (95\% CI: 13.1-18.5) and 3.5\% (95\% CI: 2.1-4.8) of children were stunted, underweight and wasted respectively. Two hundred eight (29.9\%) infants and young children fell in the poor ICFI category and $276(39.9 \%)$ were fell in to high feeding index category. Low feeding index tercile was positively and significantly associated with stunting and underweight respectively whereas high feeding index tercile was negatively and significantly associated with stunting and underweight respectively as compared to medium feeding index tercile. Children who belonged to poor feeding practice 2.4 times (AOR $=2.4$ (95 \% CI: 1.39-4.2) more likely to be underweight whereas children who belonged to good child feeding practice $55 \%$ (AOR $=0.45$ (95 \% CI: $0.27-0.75$ ) less likely to be stunted as compared to children who belonged to medium child feeding practices.

Conclusion: Child feeding practices were not optimal. Decreasing of infant and child feeding index score and increasing of stunting, underweight and wasting prevalence when the age of children increased were observed in this study reflected that infant and child feeding practices were not age appropriate. Health workers and health extension workers should educate mothers on complementary feeding with demonstration of the effect of the practice on children who optimally feed.

Keywords: Infant and Young Child Feeding Practices; Infants and Young Children Feeding Summary Index; Nutritional Status of Under Two Years Children.

\section{Introduction}

A high malnutrition rate, which is a reflection of poor feeding practices in developing countries among children under five has been consistently high due to low prevalence of exclusive breastfeeding and other inappropriate feeding practices. Lancet series 2013 suggest that under nutrition, underlies high deaths of children younger than 5 years annually worldwide. In Africa, among under five children,35.6\% are stunted, $17.7 \%$ are underweight and $8.5 \%$ are wasted in 2011[1] .However, the government of Ethiopia and other stakeholders implementing a number of strategies aimed at improving nutritional status and IYCF practices, child malnutrition is still high $(40 \%$ of under five children were stunted) [1] and the aggregate data from the year 2005-2009 estimated that $28 \%$ of all child deaths were accounted by malnutrition in Ethiopia [2].

As the report of different studies, the prevalence of stunting is high also in the regions and woredas in Ethiopia. 44\% in Southern Nation Nationalities Peoples Region, 51.4\% in Tigray 
region, 22\% in Addis Ababa, 52\% in Amhara region in under 5 children [1]. The magnitude of stunting varies significantly from woreda to woreda even in the same zone in southern Region. In Gamo Gofa Zone, among children, $18.7 \%$ were stunted in Kamba Woreda [3], 45.9\% were stunted in Arbaminch Zuria Woreda [4]. The distribution of stunting prevalence is also different in specific age groups. Study done in Sodo Zuria woreda, southern Ethiopia showed that prevalence of stunting was $16.7 \%$ for infants aged 6-8 months, 33.3\% for infants aged 9-11 months and $50 \%$ for children 12-24 months [5]. The finding of stunting prevalence in EDHS 2011 is also shows the same pattern of distribution as Sodo Zuria Woreda, 9.3\% for infants aged 6-8 months; $21.4 \%$ for infants $9-11$ months; $32 \%$ for children $12-17$ months and $47.1 \%$ for children 18-23 months [6]. In both studies, the nutritional status is getting worse when age increase. This imply that high attention should be given for young age children because stunting is associated not only with poor physical growth, but also affect cognitive abilities that are irreversible after two years of age [7, 8]. The implication of child under nutrition that has not been corrected early is extended to the entire family and subsequent generation. Malnutrition specially stunting prevents proper brain development, which means children are less able to start school when they should, and less able to learn and perform. Adults who were undernourished in childhood earn significantly less and contribute less to economic growth. Under nutrition also reduces Gross Domestic Product in every country across the globe [6].

According to the report of the Social and Economic Impact of Child Under nutrition study in Ethiopia, 67\% of the working-age population in Ethiopia is currently stunted; associated with child under nutrition, Ethiopia lost Ethiopian birr (ETB) 55.5 billion in the year 2009, which is equivalent to $16.5 \%$ of GDP and $16 \%$ of all repetitions in primary school are associated with stunting. one of the key messages in the report was "eliminating stunting in Ethiopia is a necessary step for growth and transformation"[2]. However, The critical role of feeding practices and, especially, of optimal complementary feeding practices along with continued breastfeeding among children six months and onwards to reduce young child undernutrition and mortality is well recognized $[8,10]$, Sub optimal feeding practices and inadequate intake of complementary food and which were below the WHO recommendations have been widely documented in Ethiopia.

Nationally in 2011, only $4 \%$ of children 6-23 months received a minimum acceptable diet. Among 6-23 months of age, $23.3 \%$ in Kamba woreda, $25.5 \%$ in Arbaminch woreda met minimum dietary diversity. Among children 6-23 months old, $25.8 \%$ in Kamba Woreda, $45 \%$ in Sidama Woreda met the recommended minimum meal frequency $[4,6,11,12]$. In the reports, majority of children were not met the WHO recommendations of minimum meal frequency and dietary diversity. In the cross sectional study of child feeding practices assessment using summary index done among HIV exposed infants also revealed that only $36.6 \%$ of infants fell in the good feeding practices and the majority of infants feeding practices were sub optimal [13]. Despite some improved trends in the proportion of children stunted and underweight in the last 15 year in Ethiopia, the prevalence of stunting and improper child feeding practices are still high in the country. Therefore, assessment of nutritional status and its association with infant and young child feeding summary index among 6-23 months age children in the study area in particular and Ethiopia at large is important for appropriate decision making on child nutritional interventions.

\section{Methods and Materials}

\section{Study Area and Period}

This study was conducted in Demba Gofa Woreda as of March to May 2016. Demba Gofa woreda is located $250 \mathrm{~km}$ from Arbaminch city which is zonal town of Gamo Gofa Zone and $265 \mathrm{~km}$ far from regional city of Hawassa, SNNPR. There are 38 rural kebeles in the woreda. According to Demba Gofa woreda Health Office report, the total population size of the woreda as projected to the year 2016 is 104167 of which 53125 is females and 51042 males. Sixteen thousand two hundred sixty (15.6\%) are under five. Twenty four thousand two hundred seventy one $(23.3 \%)$ are reproductive age group women. The estimated number of infants and young children (aged 6-23 months) from the whole of the woreda is 3656 . In the woreda 4 health centre and 38 health post [14]. Agriculture is the major source of income for the woreda. The major crops produced include Sorghum, Maize, Teff, Wheat, Barley, Bean, Pea, Lentil and legumes, nut and Sweat potato and enset, a root crop in the banana family [15].

\section{Study design and Population}

A community based cross sectional study was conducted in which all infant and young children aged 6 months to 23 and their mother in Demba Gofa woreda were used as source population, all infant and young children aged 6 months to 23 and their mother in the selected kebeles were used as study population moreover all randomly selected infant and young children aged 6 months to 23 and their mother in the selected kebeles of the woreda were used as sample population. The study included children 6-23 months of age where as excluded a child who had serious malformation that could affect anthropometric measurements and feeding, severely ill further more mothers who reported or obviously pregnant at the time of survey, because pregnancy affect BMI of mother.

\section{Sample Size Determination}

Emergency Nutrition Assessment (ENA) for Smart sample-size calculator was used for sample size calculation by considering a population size of 3,656 children 6 to 23 months of age in Demba Gofa Woreda [14].The prevalence of stunting was used for sample size determination based on previously published research from Sodo Zuria woreda, Wolayta Zone, SNNPR. The prevalence of stunting in specific age group was $16.6 \%$ for infants aged 6-8 months ; $33.3 \%$ for infants aged 9-11 months and 50\% for children aged 12-23 months [5]. The largest 50\% was used in the sample size calculation, a desired precision of $5 \%$ and a design effect of 2 was used, the sample size calculated was 695 [16]. The final sample size including $5 \%$ non-respondent was 730 
children mother pairs.

\section{Sampling Technique}

A multistage random sampling technique was used to select Kebeles in the first stage and eligible children in the second stage. The total Kebeles of the Woreda are 38, all are rural Kebele. Out of 38 Kebeles, 11 Kebeles were randomly selected for this study. These selected Kebeles are: Barea, Yela, Saziga Karza, Zulize Tsila, Dombe, Suka, Zatda Dolla, GaylaChalbe, Tsala Bana and Yallo Aliza. Then the number of study participants was allocated for each kebele based on proportional to population size allocation methods. Rapid censuses was conducted first to identify the target household using already existing 1 to 5 network leaders in each randomly selected Kebeles. Study units (mothers/caregiver-child pairs) were selected from each Kebele by lottery method. In one house hold, number of eligible child was more than one then one child was selected by lottery method of simple random sampling.

\section{Study variables}

\section{Dependent variable: Nutritional status of children}

Independent variables: Infant and young child feeding practices( dietary diversity, breast feeding status, meal frequency, avoiding bottle feeding, seven day food frequency score, hand washing practices),Infant and young child feeding index terciles, wealth index of the house hold, number of children in the household, Child characteristics (age, sex and mothers' verbal report on episodes of diarrheal, cough and fever of child in the last two weeks) and Maternal characteristics (age of mother; occupation of mother; educational status of mother; body mass index of mother (BMI), Antenatal and Post natal care visit, information on child feeding).

\section{Infant and Young child Feeding Index Creation}

IYCFI devised by Mary Arimond, Marie Ruel, and Purmina Menon of the International Food Policy Research Institute (IFPRI) and subsequently developed by IFPRI and the Food and Nutrition Technical Assistance (FANTA) project as a Knowledge-Practices Coverage KPC2000+[17, 18, 19] was adapted to the local context to construct cross sectional child feeding index using the current feeding recommendations. The infant and young child feeding index (ICFI) was included 7 components.

The dietary diversity score include seven food groups which are adopted from the WHO indicators for assessing infant and young child feeding practices. Mothers were asked to report all food items and beverages given to the child during the previous day of the survey. Then, all food items and beverages consumed by the child were categorized into seven food groups as (1) grains, roots, and tubers, (2) legumes and nuts, (3) dairy products, (4) flesh foods, (5) eggs, (6) vitamin-A rich fruits and vegetables, and (7) other fruits and vegetables(10). Each group was scored 1 point, if the group received by the child, if not received by the child scored 0 point and the total score of each child is 0 to 7 . Scores were assigned to reflect the age specific distributions of infants in terciles.
The seven day quasi food frequency score was a modified food group frequency and measured as "How many days in the last seven days was given [food group]?" The number of days that a food group has consumed recorded for each child with a maximum of seven days.

The list of foods summed is the same as for the 24hour diversity score, with the exception that grains have been combined with roots/tubers. In seven day food group frequency score, each food group was scored 0 if not given to the infant in the previous week, scored +1 if given one to three days, and +2 if given four or more days in the previous week. These scores were then summed to give a possible range of 0 to 14 . Based on this total score, a new score of 0-2 was assigned, reflecting the agespecific distribution.

The scoring of meal frequency was based on current feeding recommendations, according to which 6- to 8-months-old infants should receive complementary foods at least 2 to 3 times a day, 9- to 11-months-oldinfants at least 3 to 4 times a day, and 12- to 23-months-old children at least 3 to 4 times a day [17].

\section{Breast feeding and bottle use scoring}

breastfeeding received a score of 2 for infants $6-12$ months of age, a score of 1 for older children, and a score of 0 for non-breastfeeding children of any age. Avoidance of baby bottles was scored 1 (good practice), and their use received a score of 0 at any age because the practice is considered potentially harmful for all children

Scoring for hand washing practices before cooking food and before feeding child: scoring system was to assign a score of 0 for a potentially harmful practice (not washing hands) and a score of 1 for a positive (washing hands).

\section{Data Collection Instrument and Procedure}

A pre-tested structured questionnaire was used to collect maternal characteristics, household characteristics, child characteristics and feeding practices of infant and child. Questionnaire adapted from KPC 2000+ model [17] was used to collect 24 hours recall and 7 day quasi food group frequency data of infant and child feeding practices. Questionnaire adapted from EDHS 2011 [6] was used to collect socio demographic characteristics data. All the interviews and measurements were conducted at the residences of the study participants. Health professionals were recruited and trained for two days on data collection techniques. The data collection process was closely supervised and collected data were checked for completeness and inconsistencies in the field. Birth date of infant and child was obtained from their immunization card and for those who didn't have this documents, the date was obtain from mother by verbal report.

\section{Anthropometric Measurements}

A recumbent length measurement was taken by trained nurses with their respective assistants (HDA) by using wooden measuring board precise to the nearest $0.1 \mathrm{~cm}$. Two independent length measurements were taken, and the average 
was determined and was computed and expressed as z-scores by using WHO Anthrop software and was classified according to the WHO Child Growth Standards (2007).The weight of infants and children was measured by Salter scale (spring balance) to the nearest $10 \mathrm{~g}$. Mother's height and weight were measured twice. The weight was measured using electronic measuring scale (SECA) which is made from Frankfort, Germany and read to the nearest $0.1 \mathrm{~kg}$ on bare foot and with the minimum possible light clothes and height was measured using a non-stretchable measuring tape standardized against a stadiometer then BMI was calculated.

\section{Wealth Index Creation}

For determination of socioeconomic status of household, wealth index was created from the variables adapted from EDHS 2011 household questionnaire using principal components analysis. These variables included house hold goods, farm animals, main source of drinking water; type of sanitation facility and main material of the floor and of the roof. Categorical variables to be used were transformed into separate dichotomous $(0-1)$ indicators. These indicators and those that were continuous were then examined using a principal components analysis to produce a common factor score for each household. The correlation matrix for the variables contain 2 or more correlations of 0.30 or greater variables sample adequacy greater or equal to 0.50 and the Bartlett test of sphericity is statistical significancy assumptions for principal component analysis were checked. Once the index was computed, wealth terciles (from low to high) were obtained by assigning the household score to each household member.

\section{Operational definitions and definition of terms}

Child feeding index score: A summary of 7 key feeding practices scorethat vary from a minimum of 0 to a maximum of 11 for infants and young children aged 6-23 months.

Poor child feeding practice: an infant and young child's summary feeding index score belonging to lower tercile of feeding index.

Medium child feeding practice: an infant and young child's summary feeding index score belonging to middle tertcile of feeding index.

Good child feeding practices: an infant and young child's summary feeding index score belonging to higher tercile of feeding index.

Low socioeconomic status: household which belonged to lower tercile of wealth index score.

Medium socioeconomic status: household which belonged to medium tercile of wealth index score.

High socioeconomic status: household which belonged to higher tercile of wealth index score.

Stunting: length for age $<-2$ z-scores of the median WHO child growth standards.

Underweight: weight for Age $<-2 \mathrm{Z}$ score of the median WHO child growth standards
Wasting: weight for Length $<-2 \mathrm{Z}$ score of the median WHO child growth standards

\section{Data Processing and Analysis}

All data were checked for completeness, consistencies, coded and entered to Epidata 3.1 and were exported to SPSS version 20. WHO Anthrop version 3.2.2.1 software was used for nutritional status analysis. The data were checked for outliers of Z-scores which were outside the WHO flags: WLZ -5 to 5 ; LAZ -6 to 6; and WAZ -6 to 5. Descriptive statistics was computed for all continuous variables and frequency distribution was computed to evaluate the distribution of categorical variables. To assess the association between infant and child feeding practices and nutritional status in bivariate analysis, chi-square test for trends and binary logistic regression were used. The associations between the characteristics of the participants and the ICFI and also between the characteristics of the participant and the nutritional status of the child separately were tested to identify the potential confounders. For multivariable analysis, multiple logistic regressions were used after adjusting for the confounders. Multi collinearity was checked by examining the standard errors for the b coefficients (a standard error larger than 2.0. All tests was two-sided and $\mathrm{p}<0.05$ was considered for statistical significance. The internal consistency of the CFI components was measured by the Cronbach's $\alpha$ coefficient. The Cronbach's $\alpha \alpha$ value higher than 0.7 was generally considered to be satisfactory [20]. The length for age (LAZ score), weight for Age (WAZ score) and weight for height (WHZ score) were computed and compared with reference data from World Health Organization growth chart.

\section{Data Quality Control}

Questionnaires was prepared first in English by the Investigator and then translated to Amharic by another individual who is native to Amharic and is an expert in translation. The questionnaire was translated back to English by another individual in order to maintain its consistency. Data were collected by trained data collectors and pre testing of the instrument was made before the actual data collection. For this: investigator, supervisors and data collectors were a part in a pretest of the survey questionnaire among $5 \%$ of the study subjects similar to the study population in Geze Gofa woreda kebele, which is adjacent to Demba Gofa woreda kebeles, and the necessary modifications and correction were made to standardize and ensure its validity. The principal investigator and supervisors supervised the data collector on daily basis for completeness and consistence of the filled questionnaires.

\section{Results}

\section{Socio demographic Characteristics}

From 730 sample size, 696 (95.34\%) child-mother pair was participated in the study. The mean $( \pm \mathrm{SD})$ age of mothers was $28.35 \pm 4.4$ years. Among study participants, 302(43.4\%) can only read and write and majority of them $621(89.2 \%)$ were protestant. A large proportion of mothers $667(95.8 \%)$ were Gofa ethnic and $249(35.8 \%)$ mothers belonging to high wealth index 
[Table1]

Table 1: Maternal and house hold characteristics of study participants in Demba Gofa woreda 2016

\begin{tabular}{|c|c|c|}
\hline Socio-demographic characteristics & Frequency $(\mathrm{N}=696)$ & percent \\
\hline \multicolumn{3}{|l|}{ Maternal age in years } \\
\hline 15-19 years & 11 & 1.6 \\
\hline 20-24 years & 108 & 15.5 \\
\hline 25-29 years & 247 & 35.5 \\
\hline 30 and above years & 330 & 47.4 \\
\hline \multicolumn{3}{|l|}{ Religion of mother } \\
\hline Protestant & 621 & 89.2 \\
\hline Other religion of mother & 75 & 10.8 \\
\hline \multicolumn{3}{|l|}{ Ethnicity of mother } \\
\hline Gofa ethinic & 667 & 95.8 \\
\hline Other ethnics & 29 & 4.2 \\
\hline \multicolumn{3}{|l|}{ Marital status of mother } \\
\hline Married & 689 & 99.0 \\
\hline Divorced & 7 & 1.0 \\
\hline \multicolumn{3}{|l|}{ Educational status of mother } \\
\hline Illiterate & 122 & 17.5 \\
\hline Read and write & 302 & 43.4 \\
\hline Primary and above level & 272 & 39.1 \\
\hline \multicolumn{3}{|l|}{ Occupation of mother } \\
\hline House wife & 669 & 96.1 \\
\hline Other occupations & 27 & 3.9 \\
\hline \multicolumn{3}{|l|}{ Utilization of Health services } \\
\hline Mothers received ANC services & 546 & 78.4 \\
\hline Mothers received PNC services & 535 & 76.9 \\
\hline \multicolumn{3}{|l|}{ Wealth index status } \\
\hline Low SE status & 223 & 32 \\
\hline Medium SE status & 224 & 32.2 \\
\hline High SE status & 249 & 35.8 \\
\hline
\end{tabular}

The mean $( \pm$ SD) age of the children was $13.15( \pm 5.27)$ months and $369(53 \%)$ were males. In the last 2 weeks, $57(8.2 \%)$ suffered from fever, $129(18.8 \%)$ from diarrhea and $46(6.6 \%)$ from cough (Table 2).

\section{Nutritional Status of Children 6-23 Months}

Among the study population $43.8 \%$ (95\% CI: 40.1347.5)), $15.8 \%$ (95\% CI: 13.1-18.5)) and 3.5\% (95\% CI: 2.1-4.8) respectively were stunted $(<-2$ LAZ Score), wasted $(<-2$ WHZ Score), and underweight (<-2 WAZ Score) respectively (Table 3). under nutrition, of the three age groups, the highest prevalence of stunting, underweight and wasting found in 12-23 months age group children and the lowest found in 6-8 months age group children (Figure 1).

Mean Z score (mean \pm SD) of LAZ, WAZ and WHZ indices was-1.68 ( \pm 1.07$),-0.69( \pm 1.1)$ and $0.25( \pm 1.2)$ respectively for all age groups. The distribution pattern of all three indicators mean $\mathrm{Z}$ score of LAZ, WAZ and WHZ across the age groups were declining when age increase(Figure 2).

When we see infants and children age in relation to 
Table 2: Child characteristics of the study participants in Demba Gofa woreda, 2016

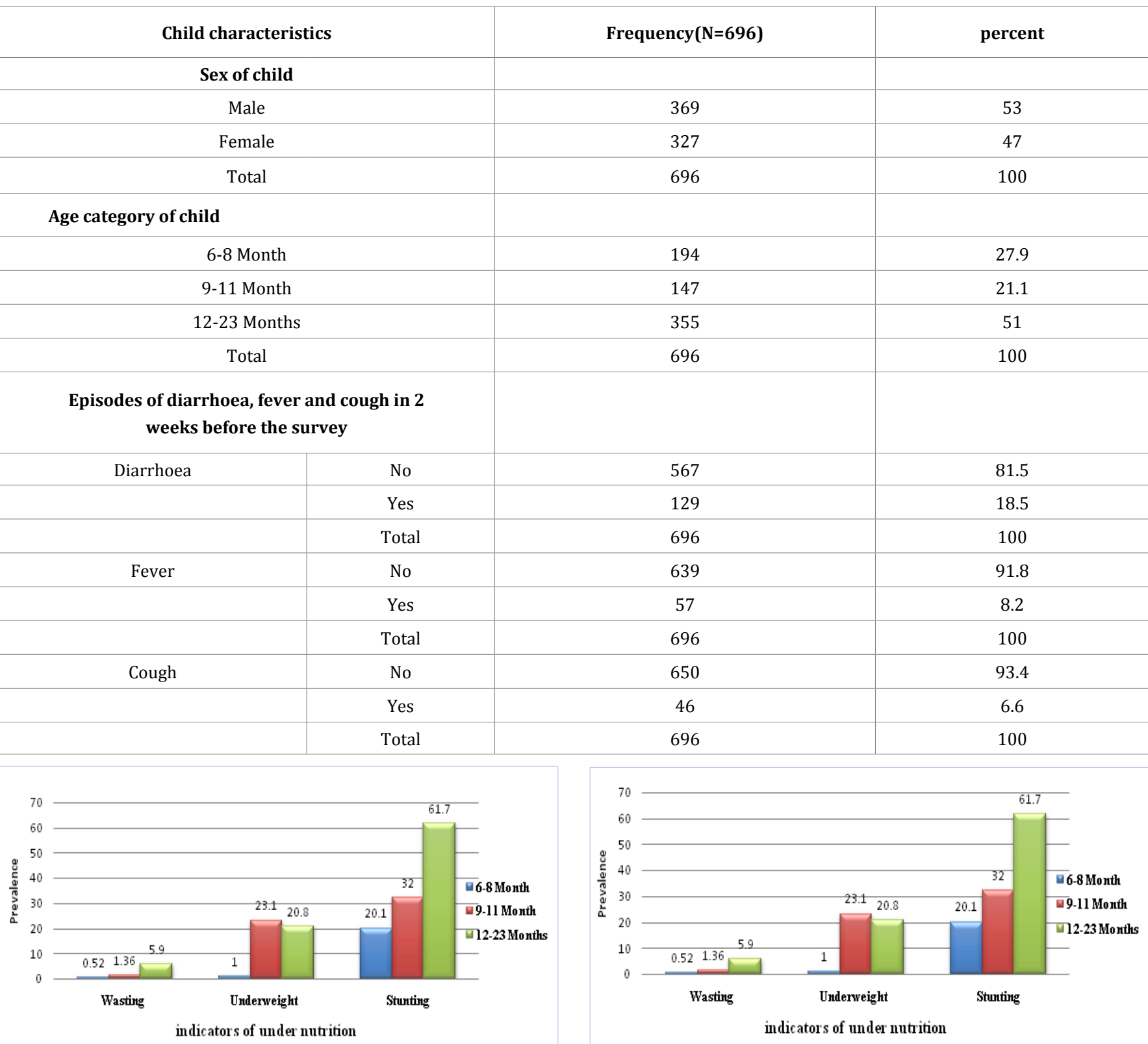

Figure 1: Infants and young children age in relation to under nutrition in Demba Gofa District, 2016

\section{Infant and Young Child Feeding Practices in Demba Gofa Woreda}

In Demba Gofa woreda breastfeeding is universal practices. However, only $443(63.6 \%)$ mothers still breast-fed at the time of interview in all age groups. About 219(31.5\%) mothers used bottles with nipples for feeding milk and other liquids.

Among all children, 97(13.9\%) children were not introduced complementary food. Forty four $(22.6 \%)$ among 6-8 months age group infants, 37(25\%) among 9-11 months age group infants and $16(4.5 \%)$ among $12-23$ month age children
Figure 2: Distribution pattern of LAZ, WAZ and WHZ mean Z score among children by age groups in Demba Gofa Woreda, 2016

were not introduced complementary food.

Proportion of infants and children obtained the highest score for the number of meal frequency that they ate in the last $24 \mathrm{hrs}$ were $148(76.3 \%), 79(53.7 \%)$ and $183(51.5 \%)$ among 6-8 months age, 9-11 months age and 12-23 months age group respectively and $140(72.2 \%), 64(43.5 \%)$ and $84(23.7 \%)$ among 6-8 months age, 9-11 months age and 12-23 months age group respectively for the number of food groups that they received in the last $24 \mathrm{hrs}$. In both meal frequency and dietary diversity score, the highest proportion was observed in 6-8 months age group. The individual combined food-frequency score for the past 
seven days ranged from 0 to 12 , but it was theoretically ranged from $0-14$. The mean \pm SD food frequency score was $4.7( \pm 2.4)$ for all infants and children. The highest mean \pm SD observed in 12-23 months age group (5.2 \pm 2.2$)$ ) and lowest observed in 6-8 months age group $(4.09 \pm 2.48)$ (Table 3 ).The food group received by the majority of children was grains, roots and tubers and the food group received by no one child was flesh foods (meat, fish, poultry and liver/organ meats) food groups in the last 24 hours and 7 days during the survey Figure 3.

\begin{tabular}{|c|c|c|c|c|c|c|}
\hline \multirow{3}{*}{ Age category of child } & \multicolumn{6}{|c|}{ Nutritional status } \\
\hline & \multicolumn{2}{|c|}{ Length for age } & \multicolumn{2}{|c|}{ Weight for length } & \multicolumn{2}{|c|}{ Weight for age } \\
\hline & Stunted & LAZ $\geq-2 S D$ & Wasted & WHZ $\geq-2 S D$ & Underweight & WAZ $\geq 2$ SD \\
\hline 6-8 Month n=194 & $39(20.1)$ & $155(79.9)$ & $1(0.5 \%)$ & $193(99.5 \%)$ & $2(1 \%)$ & 192(99\%) \\
\hline 9-11 Month $n=147$ & $47(32 \%)$ & $100(68 \%)$ & $2(1.4 \%)$ & $145(98.6 \%)$ & $34(23.1 \%)$ & $113(76.9 \%)$ \\
\hline $\begin{array}{l}\begin{array}{l}\text { 12-23 Months } \\
\mathrm{n}=355\end{array}\end{array}$ & $219(61.7 \%)$ & $136(38.3)$ & $21(6 \%)$ & $334(94 \%)$ & $74(21 \%)$ & $281(79 \%)$ \\
\hline Total $N=696$ & $305(43.8 \%)$ & $391(56.2)$ & $24(3.4 \%)$ & $672(96.6 \%)$ & $110(15.8 \%)$ & $586(84.2 \%)$ \\
\hline
\end{tabular}

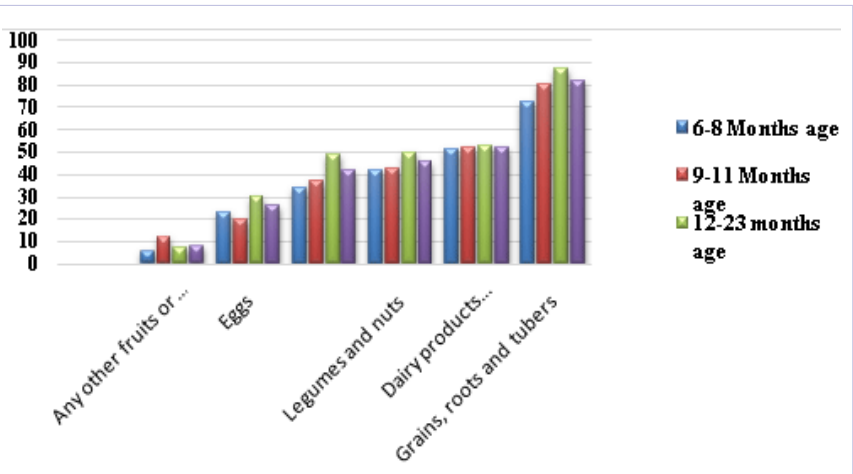

Food groups

Figure 3: Food groups given to children in the last 24 hours at survey time in Demba Gofa Woreda, 2016

The prevalence of hand washing with soap or ash before preparing food and before feeding child was $64.7 \%$ and $38.9 \%$ respectively in all mothers. In this study, not washing hands before preparing food and before feeding child were significantly associated with diarrheal disease $(\mathrm{p}<0.001)$.

\section{Distribution of Infant and Child Feeding Index}

The ICFI scores varied from the minimum 0 to the maximum10 in this sample (but a theoretical maximum was $11)$. The mean $( \pm$ SD) ICFI score of all children in the study was $6.14 \pm 2.5$. The mean \pm SD ICFI scores ranged from a low value of $5.48 \pm$ (2.54) in 9-11 months old infants to a comparatively-higher value of $6.53 \pm 2.5$ in 6-8months old infants(Table 5), which were almost half of the maximum possible score of 10 , indicating a major gap in the child feeding practices. The ICFI can be used for determining the gap between the practices being followed and the optimal practices and, hence, can be used as a screening tool at the community level. The ICFI scores were divided into low (score $\leq 4$ ), medium (score 5-7), and high (score 8-10) terciles. Among all infants and young children, 208(29.9\%), 212(30.5\%) and $276(39.6 \%)$ fell into the low, medium and high category of child feeding index respectively (Figure 4).

When we see the distribution of children by their feeding terciles in relation to age group, the highest proportion of infants and children fell in to high feeding index tercile was found in the younger age infants (6-8 months age) (Figure 5).The proportion of infants and children fell in to high child feeding index tercile among all age group as well as in each age group was below $50 \%$.

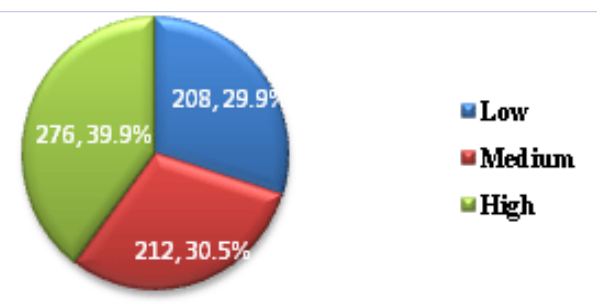

Figure 4: Distribution of children by their feeding index terciles in Demba Gofa Woreda, 2016

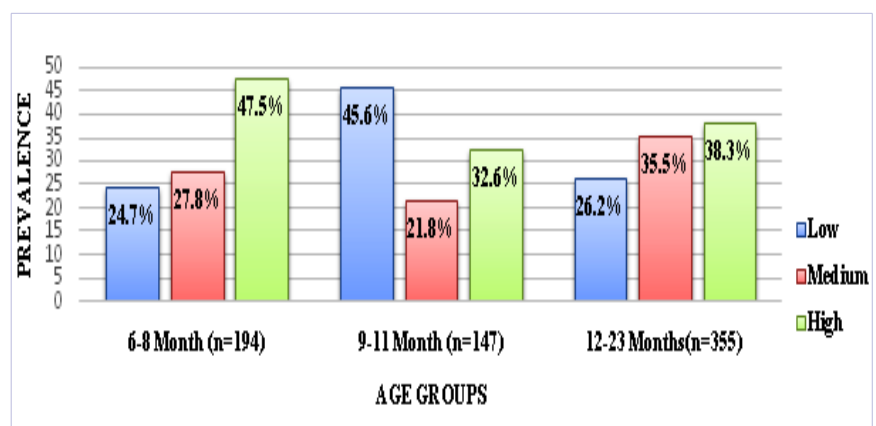

Figure 5: Distribution of children by their feeding index terciles in relation to age groups in Demba Gofa Woreda, 2016 
The mean score of micronutrient and energy source components of child feeding index were also examined. The mean age specific score of 7 days food frequency, dietary diversity, and meal frequency for 6-8 months age group was 1.6/2, 1.57/2 and $1.54 / 2$ respectively. All the three mean score were satisfactory and resulting to a considerably improved child feeding index. Whereas for age group of 9-11 months, the mean score of 7 day food frequency, dietary diversity and meal frequency were not satisfactory $(1.3 / 2,1.36 / 2$ and $1.29 / 2$ respectively), leading to a relatively low child feeding index. The same was true for age group of 12-23 month as for age group of 9-11 months except meal frequency score, $1 / 2$ for 7 days food frequency score, 1.13 for dietary diversity score, 2.26/3 for meal frequency score. For all age groups, mean value of avoiding bottle feeding was satisfactory, $0.7 / 1$ for $6-8$ months, $0.65 / 1$ for $9-11$ months, and $0.67 / 1$ for 12 23 months. Meal value of avoiding bottle feeding for 6-8 months age group was still high comparing with other age groups (Figure $6)$.

\section{Internal Consistency of Infant and Child Index}

The Cronbach's $\alpha$ a coefficient was used to estimate internal consistency of the index. For all age groups, when all 7 (seven) items of the index were included, the value of the Cronbach's $\alpha \alpha$ coefficient was 0.584 , which was below acceptable range $(<0.70)$. But, removing breast feeding from the index increased the Cronbach's $\alpha \alpha$ coefficient to the acceptable range

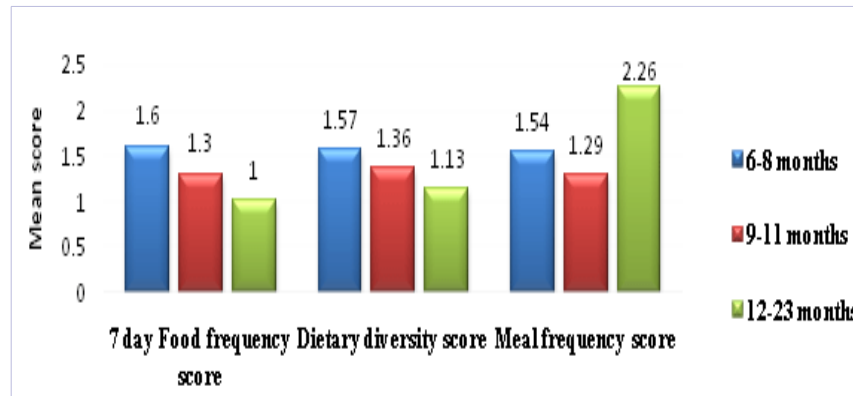

Major three components ofICFI

Figure 6: Mean score of age specific 7 days food frequency, 24hours DDS and meal frequency score by age groups

(0.705). The correlation of current breast feeding with the total summary index was also very weak (table 5) Therefore, to create composite variable of child feeding practices, current breasting score was removed from the index creation. Without current breast feeding score, the Cronbach's $\alpha \alpha$ coefficient of this study was good for the three age groups. For 6-8 months of age 0.744 (95\% CI: 0.684-0.797); for 9-11 months of age 0.778 (95\% CI: 0.717-0.829); for 12-23 months of age 0.738 (95\% CI: $0.693-$ 0.778).The ICFI showed positive and strong correlation with seven day food group frequency, 24 hour meal frequency score, and 24 hour food diversity score [Table 5].

Table 4: Results of Key child feeding practices/ indicators in Demba Gofa woreda, 2016

\begin{tabular}{|c|c|c|c|c|c|}
\hline \multirow{2}{*}{\multicolumn{2}{|c|}{ Feeding practices }} & \multirow{2}{*}{$\begin{array}{c}\text { All age } \\
(\mathrm{N}=696) \\
\text { 6-8 Month } \\
(\mathrm{n}=194)\end{array}$} & \multicolumn{3}{|c|}{ Age category of child } \\
\hline & & & $\begin{array}{l}\text { 9-11 Month } \\
(\mathrm{n}=147)\end{array}$ & $\begin{array}{l}\text { 12-23 Months } \\
(\mathrm{n}=355)\end{array}$ & \\
\hline \multicolumn{2}{|c|}{$\begin{array}{l}\text { Number of children received Legumes and nuts in the last } 24 \\
\text { hours }\end{array}$} & $320(46 \%)$ & $81(41.8 \%)$ & $62(42.2 \%)$ & $177(49.9 \%)$ \\
\hline \multicolumn{2}{|c|}{ Number of children received Egg in the last 24 hours } & $179(25.7)$ & $45(23.2 \%)$ & $29(19.7 \%)$ & $105(29.6 \%)$ \\
\hline \multicolumn{2}{|c|}{$\begin{array}{l}\text { Number of children received flesh foods (meat, fish, poultry and } \\
\text { liver/organ meats) eaten in the last } 24 \mathrm{hrs}\end{array}$} & $0(0 \%)$ & $0(0 \%)$ & $0(0 \%)$ & $0(0 \%)$ \\
\hline \multicolumn{2}{|c|}{ Number of children avoided Bottle feeding } & $477(68.5)$ & $143(73.7 \%)$ & $96(65.3 \%)$ & $238(67.1 \%)$ \\
\hline \multicolumn{2}{|c|}{$\begin{array}{l}\text { Number of children received dairy products (milk, yogurt } \\
\text { cheese) in the last } 24 \text { hours }\end{array}$} & $364(52.3)$ & $100(51.5 \%)$ & $76(51.7 \%)$ & $188(52.9 \%)$ \\
\hline \multicolumn{2}{|c|}{$\begin{array}{l}\text { Number of children received grains, rooter and tuber in the last } \\
\qquad 24 \text { hours }\end{array}$} & $570(81.9)$ & $141(72.7 \%)$ & $118(80.3 \%)$ & $311(87.6 \%)$ \\
\hline \multicolumn{2}{|c|}{$\begin{array}{l}\text { Number of children received Vitamin A rich fruits and } \\
\text { vegetables in the last } 24 \text { hours }\end{array}$} & $292(42 \%$ & $66(34 \%)$ & $54(36.7 \%)$ & $172(48.5 \%)$ \\
\hline Mean of dietary diversity score & $\begin{array}{l}\text { No of food } \\
\text { groups }\end{array}$ & 2.55 & 2.29 & 2.41 & 2.75 \\
\hline Children not introduced Complementary food & $\begin{array}{l}\text { No of food } \\
\text { children }\end{array}$ & $97(13.9 \%)$ & $44(22.6 \%)$ & $37(25.1 \%)$ & $16(4.5 \%)$ \\
\hline
\end{tabular}




\begin{tabular}{|c|c|c|c|c|c|}
\hline \multirow{2}{*}{$\begin{array}{l}\text { Proportion of infants and children obtained the } \\
\text { highest score for the number of meal frequency }\end{array}$} & No of meal & & $>=2$ meals & $>=3$ meals & $>=4$ meals \\
\hline & Percent & $410(58.9)$ & $148(76.3 \%)$ & $79(53.7 \%)$ & $183(51.5 \%)$ \\
\hline \multirow{2}{*}{$\begin{array}{l}\text { Proportion of infants and children obtained the } \\
\text { highest score for the number of dietary diversity } \\
\text { score }\end{array}$} & $\begin{array}{l}\text { No of food } \\
\text { groups }\end{array}$ & & $\geq 2$ food groups & $\geq 3$ food groups & $\geq 4$ food groups \\
\hline & Percent & $288(41.4)$ & $140(72.2 \%)$ & $64(43.5)$ & $84(23.7 \%)$ \\
\hline $\begin{array}{l}\text { Hand washing with soap/ash before preparing } \\
\text { food }\end{array}$ & Yes & $450(64.7)$ & $130(67 \%)$ & $78(53 \%)$ & $242(68.2 \%)$ \\
\hline $\begin{array}{l}\text { Hand washing with soap/ash before feeding the } \\
\text { child }\end{array}$ & Yes & 271(38.9) & $79(40.7 \%)$ & $51(34.7 \%)$ & $141(39.7 \%)$ \\
\hline Mean of Meal frequency in the last 24 hours & Meal number & 3.2 & 2.9 & 2.78 & 3.5 \\
\hline $\begin{array}{l}\text { Mean of seven days food frequency score(not } \\
\text { age specific score) }\end{array}$ & $\operatorname{Mean}( \pm \mathrm{SD})$ & $4.7( \pm 2.4)$ & $4.09( \pm 2.48)$ & $4.3( \pm 2.61)$ & $5.2( \pm 2.2))$ \\
\hline Mean of child feeding index & $\operatorname{Mean}( \pm \mathrm{SD})$ & $6.14( \pm 2.5)$ & $6.53( \pm 2.5)$ & $5.48( \pm 2.5)$ & $6.19( \pm 2.5)$ \\
\hline
\end{tabular}

Table 5: Internal consistency of IYCFI and its correlation with IYCFI components among infants and children Demba Gofa District in, South Ethiopia, 2016

\begin{tabular}{|c|c|c|c|c|c|c|c|c|}
\hline \multirow{3}{*}{$\begin{array}{l}\text { Components of } \\
\text { ICFI }\end{array}$} & \multicolumn{2}{|c|}{ All $(\mathrm{N}=696)$} & $\begin{array}{c}\text { 6-8 } \\
\text { Months(n=194) }\end{array}$ & & $\begin{array}{l}\text { 9-11 Months } \\
(\mathrm{n}=147)\end{array}$ & & \multicolumn{2}{|c|}{$12-23$ months $(\mathrm{n}=355)$} \\
\hline & \multirow{2}{*}{$\begin{array}{l}\text { correlation } \\
\text { of each } \\
\text { component } \\
\text { with IYCFI }\end{array}$} & \multirow{2}{*}{$\begin{array}{c}\text { Cronbach's } \alpha \\
\text { value when all } \\
\text { items included= } \\
0.584 \\
\text { Cronbach's } \\
\text { Alpha if Item } \\
\text { Deleted }\end{array}$} & \multicolumn{2}{|c|}{$\begin{array}{l}\text { Cronbach's } \alpha \text { value when all } \\
\text { items included }=0.58\end{array}$} & \multicolumn{2}{|c|}{$\begin{array}{l}\text { Cronbach's } \alpha \text { value when all } \\
\text { items included }=0.562\end{array}$} & \multicolumn{2}{|c|}{$\begin{array}{l}\text { Cronbach's } \alpha \text { value when } \\
\text { all items included }=0.694\end{array}$} \\
\hline & & & $\begin{array}{c}\text { correlation of each } \\
\text { component with } \\
\text { IYCFI }\end{array}$ & $\begin{array}{l}\text { Cronbach's } \\
\text { Alpha } \\
\text { if Item } \\
\text { Deleted }\end{array}$ & $\begin{array}{l}\text { correlation } \\
\text { of each } \\
\text { component } \\
\text { with IYCFI }\end{array}$ & $\begin{array}{l}\text { Cronbach's } \\
\text { Alpha } \\
\text { if Item } \\
\text { Deleted }\end{array}$ & $\begin{array}{c}\text { Correlation } \\
\text { of each } \\
\text { component } \\
\text { with IYCFI }\end{array}$ & $\begin{array}{c}\text { Cronbach's } \\
\text { Alpha } \\
\text { if Item } \\
\text { Deleted }\end{array}$ \\
\hline $\begin{array}{l}\text { Age specific } \\
\text { score of hand } \\
\text { washing before } \\
\text { feeding child }\end{array}$ & 0.525 & 0.531 & 0.336 & 0.584 & 0.635 & 0.47 & 0.583 & 0.645 \\
\hline $\begin{array}{l}\text { Age specific } \\
\text { score of hand } \\
\text { washing before } \\
\text { cooking food }\end{array}$ & 0.666 & 0.508 & 0.587 & 0.523 & 0.73 & 0.478 & 0.674 & 0.631 \\
\hline $\begin{array}{c}\text { Age specific } \\
\text { score of avoiding } \\
\text { bottle feeding }\end{array}$ & 0.39 & 0.564 & 0.319 & 0.582 & 0.292 & 0.551 & 0.462 & 0.681 \\
\hline $\begin{array}{l}\text { Age specific Meal } \\
\text { frequency score }\end{array}$ & 0.751 & 0.563 & 0.865 & 0.371 & 0.806 & 0.443 & 0.805 & 0.631 \\
\hline $\begin{array}{l}\text { Age specific } \\
\text { dietary diversity } \\
\text { score }\end{array}$ & 0.712 & 0.462 & 0.872 & 0.378 & 0.788 & 0.43 & 0.64 & 0.648 \\
\hline $\begin{array}{l}\text { Age specific } \\
7 \text { days food } \\
\text { frequency score }\end{array}$ & 0.754 & 0.432 & 0.802 & 0.436 & 0.825 & 0.389 & 0.77 & 0.616 \\
\hline $\begin{array}{l}\text { Age specific } \\
\text { Breast feeding } \\
\text { score }\end{array}$ & -0.096 & 0.705 & -0.204 & 0.744 & -0.233 & 0.778 & 0.02 & 0.738 \\
\hline
\end{tabular}




\section{The association between Nutritional status and Infant and Young Child Feeding Index terciles}

The analyses of chi-square test for trends between feeding index terciles and stunting, underweight and wasting were done. The association between child feeding index terciles and stunting, underweight and wasting were significant $(\mathrm{P}<0.001)$. The figure 7 shows relationship between the prevalence of Stunting, Underweight, and Wasting, and the IYCFI terciles for the children. The feeding index terciles are relates more consistently to outcomes in the three nutritional status indicators.

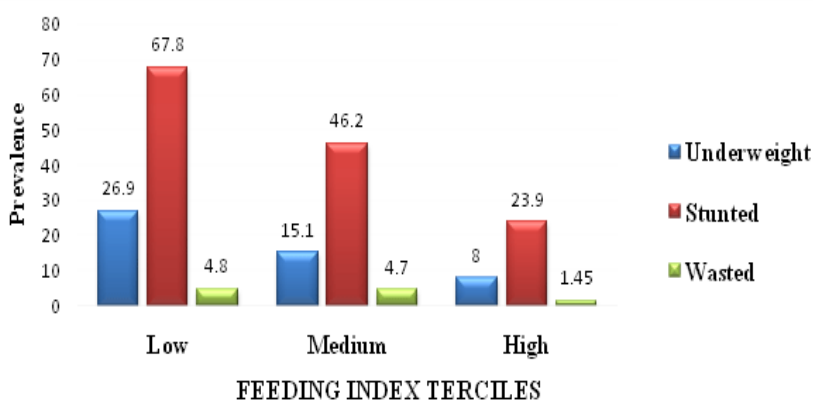

Figure 7: The relationship between underweight, stunting and wasting prevalence and IYCFI terciles in Demba Gofa Woreda, 2016
The association of high child feeding index tercile and low child feeding index tercile with stunting, underweight and wasting respectively examined by multiple logistic regression.

After controlled for potential confounders, Low feeding index tercile was positively and significantly associated with stunting and underweight respectively whereas high feeding index tercile was negatively and significantly associated with stunting and underweight respectively as compared to medium feeding index tercile. There was no any association between wasting and infant and child feeding index terciles (Table 6-8).

However, many factors in bivariate analysis were significantly associated with under-nutrition, few were significantly associated with under nutrition in multivariable analysis. Mothers did not attend any formal school and being older age child were positively and significantly associated with stunting whereas mothers who had more frequent visiting of PNC clinic was significantly reduce the odds of stunting. Being male child, older age child (12-23 months age) and high number of under five children in the house were significantly associated with underweight. Diarrhoea and high number of under five children in the house were positively and significantly associated with wasting (Table 6-8).

Table 6: Bivariate and multivariable logistic regression model predicting the likelihood of being stunting among children Stunting

\begin{tabular}{|c|c|c|c|c|c|c|}
\hline \multicolumn{2}{|c|}{ Independent Variables } & \multirow{2}{*}{$\begin{array}{c}\text { Yes } \\
39\end{array}$} & \multirow{2}{*}{$\begin{array}{c}\text { No } \\
155\end{array}$} & \multirow{2}{*}{$\begin{array}{c}\text { COR } \\
1\end{array}$} & \multirow{2}{*}{$\begin{array}{c}\text { AOR(95\% C.I. for } \\
\text { AOR) } \\
1\end{array}$} & \multirow[t]{2}{*}{ P. value } \\
\hline \multirow{3}{*}{ Age category of child } & 6-8 Month (Reference) & & & & & \\
\hline & 9-11 Month & 47 & 100 & $1.9^{*}$ & $1.2(0.6-2.2)$ & 0.659 \\
\hline & 12-23 Months & 219 & 136 & $6.4^{*}$ & $10.5(6.0-18.3)$ & $<0.000 .1$ \\
\hline \multirow{2}{*}{ Ethnicity of mother } & $\begin{array}{l}\text { Other ethnic } \\
\text { (Reference) }\end{array}$ & 8 & 21 & 1 & 1 & \\
\hline & Gofa ethnic & 297 & 370 & 2.1 & $0.99(0.34-2.9)$ & 0.996 \\
\hline \multirow{2}{*}{ Religion of mother } & Other religion (Reference) & 28 & 47 & 1 & 1 & \\
\hline & Protestant & 277 & 344 & 1.4 & $1.1(0.53-2.26)$ & 0.798 \\
\hline \multirow{2}{*}{ Maternal age } & $\geq 20$ years (Reference) & 271 & 364 & 1 & 1 & \\
\hline & maternal age $15-19$ years & 34 & 27 & 1.7 & $0.72(0.33-1.5)$ & 0.397 \\
\hline \multirow{2}{*}{ house wife } & Other occupation (Reference) & 5 & 22 & 1 & 1 & \\
\hline & House wife & 300 & 369 & $3.6^{*}$ & $3.1(0.9-10.9)$ & 0.08 \\
\hline \multirow{3}{*}{$\begin{array}{c}\text { Educational status of } \\
\text { husband }\end{array}$} & Illiterate & 22 & 19 & 1.97 & $7.6(3.02-19.2)$ & $<0.000 .1$ \\
\hline & Read and write & 133 & 117 & 1.9 & $1.7(1.039-2.8)$ & $0.035^{*}$ \\
\hline & $\begin{array}{l}\text { Primary School and above } \\
\text { (Reference) }\end{array}$ & 150 & 255 & 1 & 1 & \\
\hline
\end{tabular}




\begin{tabular}{|c|c|c|c|c|c|c|}
\hline \multirow{3}{*}{$\begin{array}{l}\text { Educational status of } \\
\text { Mother }\end{array}$} & Illiterate & 67 & 55 & 2.1 & $1(0.514-1.9)$ & 0.999 \\
\hline & Read and write & 138 & 164 & 1.45 & $1.7(0.99-2.9)$ & 0.051 \\
\hline & $\begin{array}{l}\text { Primary School and above } \\
\text { (Reference) }\end{array}$ & 100 & 172 & 1 & 1 & \\
\hline \multirow{2}{*}{ The child still breastfeeding } & No & 128 & 125 & $1.54^{*}$ & $1.5(0.99-2.4)$ & 0.055 \\
\hline & Yes (Reference) & 177 & 266 & 1 & 1 & \\
\hline \multirow{3}{*}{ Child Feeding Index terciles } & Low child feeding index & 141 & 67 & $2.4^{*}$ & $3.3(1.9-5.8)$ & $<0.000 .1$ \\
\hline & $\begin{array}{l}\text { Average child feeding index } \\
\text { (Reference) }\end{array}$ & 98 & 114 & 1 & 1 & \\
\hline & Good child feeding & 66 & 210 & $0.4^{*}$ & $0.45(0.27-0.75)$ & $0.002^{*}$ \\
\hline \multicolumn{2}{|c|}{$\begin{array}{l}\text { Number of }<5 \text { years children in the house (mean } \pm S D)(1.74 \\
\qquad \pm 0.5)\end{array}$} & 305 & 391 & 1.2 & $0.76(0.49-1.2)$ & 0.219 \\
\hline \multicolumn{2}{|c|}{ Number of PNC visit $($ mean $\pm S D)(1.85 \pm 1.2)$} & 305 & 391 & $0.4^{*}$ & $0.38(0.31-0.47)$ & $<0.000 .1$ \\
\hline
\end{tabular}

Table 7: Bivariate and multivariable logistic regression model predicting the likelihood of being underweight among children

\begin{tabular}{|c|c|c|c|c|c|c|}
\hline \multicolumn{7}{|c|}{ Underweight } \\
\hline & & Yes & No & COR & $\begin{array}{l}\text { AOR( } 95 \% \text { C.I. } \\
\text { for AOR) }\end{array}$ & P. value \\
\hline \multirow[t]{3}{*}{$\begin{array}{l}\text { Child Feeding Index } \\
\text { terciles }\end{array}$} & Low child feeding index & 56 & 152 & $2.1^{*}$ & $2.4(1.39-4.2)$ & $0.002^{*}$ \\
\hline & $\begin{array}{l}\text { Average child feeding index } \\
\text { (Reference) }\end{array}$ & 32 & 180 & 1 & & \\
\hline & Good child feeding & 22 & 254 & $0.5^{*}$ & $\begin{array}{l}0.42(0.23- \\
0.78)\end{array}$ & $0.006^{*}$ \\
\hline \multirow[t]{3}{*}{$\begin{array}{l}\text { Educational status of } \\
\text { Mother }\end{array}$} & Illiterate & 10 & 112 & 0.85 & $\begin{array}{l}0.57(0.25- \\
1.3)\end{array}$ & 0.167 \\
\hline & Read and write & 74 & 228 & 3.1 & $2.6(1.5-4.4)$ & $0.001^{*}$ \\
\hline & $\begin{array}{l}\text { Primary School and } \\
\text { above(Reference) }\end{array}$ & 26 & 246 & 1 & 1 & \\
\hline \multirow[t]{2}{*}{ Age of child } & $\begin{array}{l}\text { 6-11 month age } \\
\text { (Reference) }\end{array}$ & 36 & 305 & 1 & 1 & \\
\hline & 12-23 month age & 74 & 281 & $2.2^{*}$ & $\begin{array}{l}2.04(1.26- \\
3.3)\end{array}$ & $0.004^{*}$ \\
\hline \multirow[t]{2}{*}{ Sex of child } & Male & 71 & 298 & 1.8 & $\begin{array}{l}1.63(1.01- \\
2.6)\end{array}$ & $0.042^{*}$ \\
\hline & Female (Reference) & 39 & 288 & 1 & 1 & \\
\hline \multicolumn{2}{|c|}{$\begin{array}{l}\text { Number of }<5 \text { children in the house }(\text { mean } \pm \text { SD }) \\
\qquad(1.74 \pm 0.5)\end{array}$} & 110 & 586 & $4.8^{*}$ & $3.6(2.03-6.4)$ & $<0.000 .1$ \\
\hline \multicolumn{2}{|c|}{ Number of PNC visits (mean \pm SD) } & 110 & 586 & $0.8^{*}$ & $\begin{array}{l}0.95(0.78- \\
1.2)\end{array}$ & 0.598 \\
\hline
\end{tabular}


Table 8: Bivariate and multivariable logistic regression model predicting the likelihood of being wasting among children

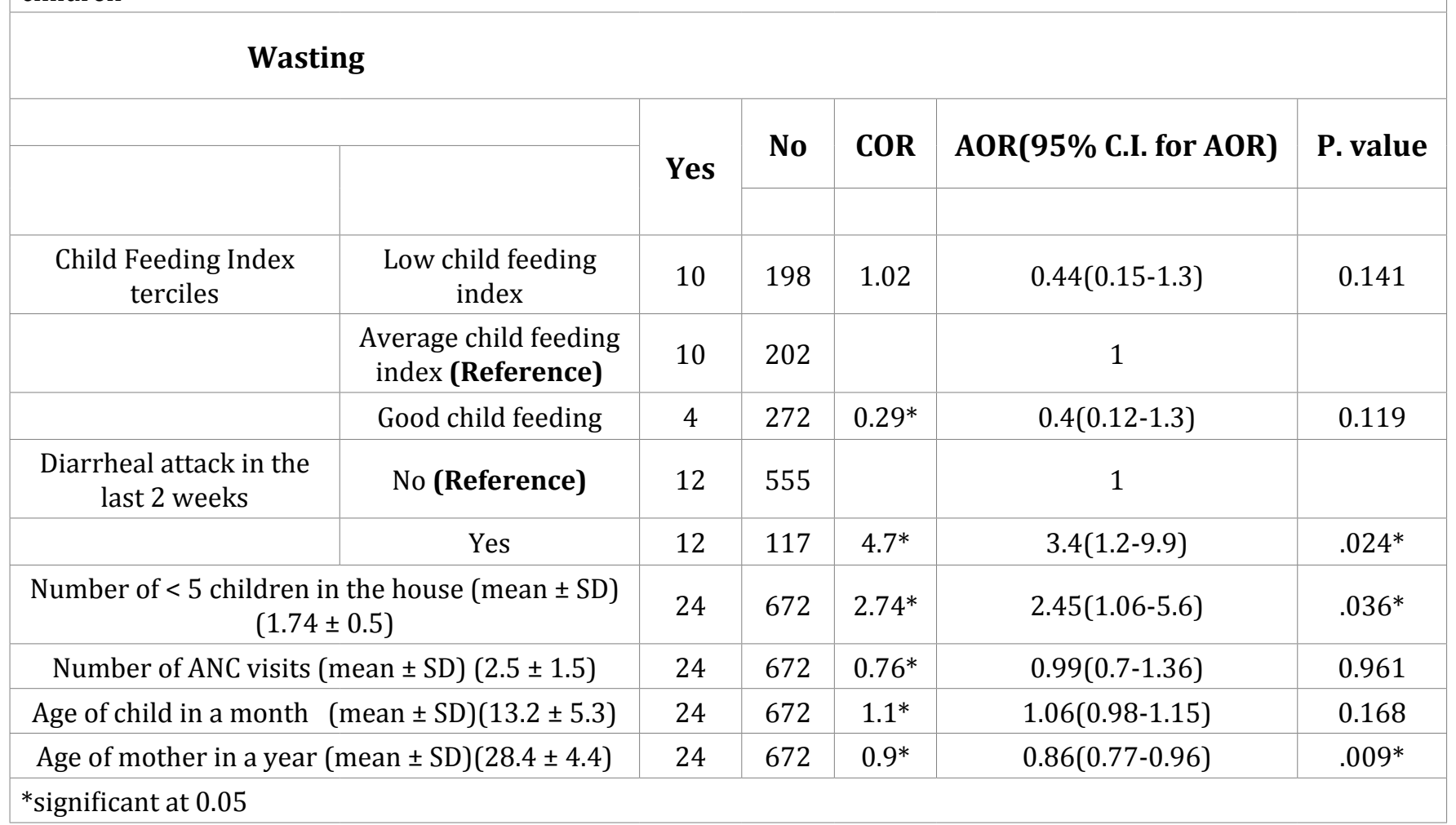

\section{Discussion}

This study has assessed nutritional status; infants and young children feeding practices using a summary index among 6-23 months age children and examined the association between nutritional status and IYCF summary index.

The prevalence of stunting (43.8\%) was high in the sample as compared to national prevalence (40\%) and nearly equal to SNNPR's prevalence $(44.1 \%)$. Butunder weight and wasting prevalence $(15.8 \%$ and $3.45 \%)$ respectively were low as compared to the national and SNNPR. The distribution of all three indicators of under nutrition were getting worse when age increase. Which were similar findings as the previous studies in our country $[1,5]$.

To assess child feeding practices by summary index of key infant and child feeding practices (composite variable), internal consistency of seven items (components) was checked. For all age groups, when all 7 (seven) items of the index were included in the reliability analysis, the value of the Cronbach's $\alpha$ coefficient was 0.584 . Which was below acceptable range $(\leq 0.70)$ [21]. But removing current breast feeding from the reliability analysis increased the Cronbach's $\alpha$ coefficient to the acceptable range, which was 0.705 . The correlation of current breast feeding with the total summary index for all age groups as well as for each age group was also very weak and negative [Table5]. Therefore, as mentioned earlier in the result part, to create child feeding index, current breasting score was removed from the index creation and treated as another independent variable in the model. The correlation of dietary diversity, meal frequency and 7 days food frequency were strong and positive for 6-8months and 9-11 months age infants, whereas for 12-23 months age children only meal frequency and 7 days food frequency were strongly and positively correlated, dietary diversity was moderately correlated for this age group. This means that the number of food groups given to a child was not increasing while the age of children increase.

The mean IYCFI score for 6-8 months age-group (6.53) was the highest as compared to other age groups whereas mean IYCFI score for 9--11 months old infants (5.48) was the lowest scores. In general, for all age groups, mean child feeing index score is not satisfactory because it is almost half of the maximum value of ten (10) [table5]. This indicates more gaps in the child feeding practices among all age groups. Decreasing of IYCFI score when age increase in the present study is not consistent with the findings of the study done in rural India which reported child-feeding index score increase with age increase [22]. The reason of inconsistency might be, majority of 6-8 months age children introduced complementary food as compared to the Indian infants. Only nine infants started complementary food at 6 months of their age. In this study, however, majority of infants started complementary food; it was not adequate when the age of children increase. Black et al. reported that even with optimum breastfeeding, children will become stunted if they do not receive an adequate quantity and quality of complementary foods after 
six months of age [8]. Our findings are in line with the report by Black et al. The micronutrient and energy source components of the index, i.e. seven days food frequency, dietary diversity and meal frequency mean score for 9-11 month and 12-23 months age groups were relatively poor whereas the stunting, underweight and wasting prevalence were high among them.

The distribution of children by their feeding index terciles for the children was $29.9 \%$ of children fell in to poor infant and child feeding practice while $39.9 \%$ of them were fell in to good infant and child feeding practice [figure6]. The result reflected that feeding practices of the majority mothers were not optimal for the children. The analysis of feeding index terciles in relation to age groups showed that most of the infants $92(47.5 \%)$ in the youngest age group (6-8 months) were found in the good infant and child feeding practice and $93(26.2 \%)$ of children among the oldest age group (12-23 months age) were found in the poor infant and child feeding practice [figure7]. This high prevalence of poor feeding practices for 9-11 months and 1223 months age group may be attributed to the high prevalence of under-nutrition in these age groups. The result of multiple logistic regression analysis of this study also showed that being older age (12-23 months) was significantly associated with stunting and underweight $(\mathrm{P}<0.001)$.

In sum, in the current study, even though breast feeding practice removed from the index creation because of its internal inconsistency with other components, the younger children (6-8 months) were more likely to be fed better than the older ones (12-23 months), and this may be because they are perceived to be very vulnerable or weak and therefore in need of more attention compared to older children who may be regarded as strong enough to feed on the adult diet and according to adult meal frequency.

In the sampled children of this study, the complementary feeding practices which were identified to be undesirable were bottle feeding practice, no introduction of complementary feeding for few children in each age group, and no feeding of flesh food group (meat, poultry, and organ meats) to their children. These undesirable feeding practices also contributed to the low mean score of infant and child feeding index.

In this study, the association between feeding index terciles and stunting and underweight by multivariate logistic regression analysis after adjusting the potential confounders showed that low feeding index tercile and high feeding index tercile were positively and negatively respectively associated with both stunting and underweight as compared to medium feeding index [table7-8]. There was no any association between wasting and feeding index terciles in this study [table 8].Wasting prevalence was very low in this study; it might be the reason for no association with IYCFI. Studies in Ethiopia, Latin America and Burkina Faso reported that infant and child feeding index was a predictor of HAZ and WAZ score [13, 23, 24, 25] $(23,24,25,13)$. The studies done in India, Garg et al. reported association between child feeding index score and under nutrition [22] whereas Srivastava et al. failed to find so [26].
The result of current study showed that children belonged to poor feeding practice (low feeding index tercile) 2.4 times more likely to be underweighted as compared to children belonged to medium feeding practice (medium feeding index tercile) whereas children belonged to good child feeding practice (high feeding index tercile) 55\% less likely to be stunted as compared to children belonged to medium child feeding practices. This indicated that the composite of feeding practices improve the nutritional status of children. This finding is similar with the study done in West Bengal, India, the finding on that study was Per unit increase in standardized IYCF score, under nutrition by all three indicators was likely to be reduced by $2-3 \%$ and the association was significant after adjustment for the potential confounders [27]. The finding of this study contradicts the findings of a study in urban settings of Ethiopia [28]where the composite index was unable to show an impact on nutritional status in the multivariate model.

However, many factors in bivariate analysis were significantly associated with under-nutrition; few were significantly associated with under nutrition in multivariate analysis.

The result of the present study indicated that child's age is determinant factors of stunting and the risk of stunting increases with age. The finding of this study showed that children in the age group12-23 months were at significantly higher risk of stunting compared with children in the youngest age category ( $\leq$ 8 months). This result is consistent with other studies in Ethiopia $[1,6,29]$. This needs attention since particularly stunting is associated not only with poor physical growth but also affects cognitive abilities that are irreversible after 2 years of age. For nutritional interventions also, children younger than 24 months of age responded much more rapidly to the improvement than older children. After a child reaches 2 years of age, it is very difficult to reverse stunting that has occurred earlier [7].

Mothers did not attend any formal school was positively and significantly associated with stunting as compared to mothers attended formal education whereas mothers who had more frequent visit of PNC clinic was significantly reduce the odds of being stunting. This result is consistent with the studies done in Arbaminch and Kamba Woreda Southern Ethiopia [3, 4]

Being male child, older age child (12-23 months age) and high number of under five children in the house were significantly associated with underweight.

In this study, a high prevalence of wasting was observed among children who had diarrhea in the two weeks before the survey as compared to children who had no diarrhea and its incidence is positively associated with wasting. This result is consistent with the studies done in West Gojam Zone and Somali region of our country, Ethiopia[29,30]. High number of under five children in the house were also positively and significantly associated with wasting as compared to low number of children.

In general, a number of interrelated maternal, child and household characteristics and morbidities of diarrhea, cough 
and fever were known to be associated with feeding practices and nutritional status of 6-23 months age children. Significant negative association of good infant and child feeding practice and significant positive association of poor infant and young child practice with undernutrition particularly stunting and underweight among 6-23 months age children after adjusting for other related factors highlighted their crucial role in child nutrition.

\section{Conclusion}

The prevalence of undernutrition particularly stunting was high among 6-23 months age children and good or optimal feeding practices, as estimated by ICFI, was not satisfactory (only $39.9 \%$ ) among the study participants. This means that only 4 out of every ten children in our study received good feeding. The advantage of composite index was that it was age-specific and could capture multiple key dimensions of IYCF practices considering possible combined influence. In the current study, children who belonged to poor child feeding practices were significantly stunted and underweight as compared to children who belonged to medium child feeding practice whereas children belonged to good child feeding practices has better nutritional status as compared to children belonged to medium child feeding practices. Decreasing of ICFI score and increasing of stunting, underweight and wasting prevalence when the age of children increased in this study reflected that infant and child feeding practices were not age appropriate and low attention have been given when the age of children increased.

\section{Recommendation}

This study showed that the combination of feeding practices significantly reduced the occurrence of under nutrition among children.

Therefore, improving of all feeding practices simultaneously is crucial to tackle the markedly increasing under nutrition rate when the age of children increased. Decision makers and program implementers had better evaluate the nutrition interventions that are being carried out at grass root level as IYCF guidelines. Demba Gofa District health office and other stakeholders should strengthen Behavior Change Communication (BCC) on complementary feeding practices Health workers and health extension workers should educate mothers on complementary feeding by emphasizing the importance of increasing consistency and variety of food, increasing meal frequency when the of age child increase

First, I would like to thank the Almighty God for providing me with good health and wisdom to be able to go through my MSc. Study successfully. Secondly, I would like to thank my advisors, Mr. Dessalegn Tamiru and Mr. Amanuel Tesfay, for their invaluable guidance and advises in all aspect of my thesis work. Thirdly, I would like to thank Demba Gofa District administration office and health sector for their provision of full time for MSc study and financial supports. Fourthly, I would like to thank all study participants for their full participation and time for this study.

\section{Strength of the Study}

It was a community-based assessment with almost 95.34\% response rate and aimed at assessing nutritional status of infants and young children and mothers real IYCF practices while visiting in their own natural environment.

\section{Limitation of the Study.}

During the study, we collected information on the feeding practices of children for the last 24 hours and for the previous one week. This might be influenced by some recall bias. The study employed a cross-sectional study design which could not establish a cause and effect relationship between the dependent and independent variables.

\section{Ethical Consideration}

Ethical clearance was obtained from ethical committee of College of Public Health; Jimma University. A formal letter, written from the college of Public Health of Jimma University, was submitted to Demba Gofa woreda health office and the woreda administration office to obtain their co-operation. Then permission and support letter were written to each respective Keble's. The purpose of the study was explained to the study subjects. At the time of data collection, a verbal consent was taken from the participants to confirm whether they were willing to participate. Those not willing to participate were given the right to do so. Confidentiality of responses was also ensured throughout the research process.

\section{References}

1. Central Statistical Agency[Ethiopia]. Ethiopia Mini Demographic and Health Survey. Addis Ababa, Ethiopia; 2014.

2. UNICEF. The cost of hunger in Ethiopia. The social and economic impact of child undernourishment in Ethiopia summary report. Addis Ababa. 2014.

3. Agedew E, Chane T. Prevalence of Stunting among Children Aged 6-23 Months in Kemba Woreda, Southern Ethiopia: A Community Based Cross-Sectional Study. Adv Public Heal. 2015;2015.

4 Jisha H, Tamiru D. Dietary Patterns and Anthropometric Status of UnderFive Children in Arba Minch Zuria, Gamo Gofa Zone: CommunityBased Cross-Sectional Study. J Adv Dairy Res. 2015;3(138). DOI: 10.4172/2329-888X.1000138

5. Moshago D, Gibson RS, Abebe Y. Assessment of the Nutritional Adequacy of Complementary Foods and Infant and Young Child Feeding Practices in Sodo Zurea District, Wolayita, Southern Ethiopia. 2016;30(Supp 1).

6. Central Statistical Agency [Ethiopia] and ICF International. Ethiopia Demographic and Health Survey 2011. Addis Ababa, Ethiopia and Calverton, Maryland, USA: Central Statistical Agency and ICF International. 2012

7. Martorell R, Khan LK, Schroeder DG. Reversibility of stunting: epidemiological findings in children from developing countries. Eur J Clin Nutr. 1994;48(1):S45-57.

8. Black RE, Allen LH, Bhutta ZA, Caulfiel LE, Onis M de, Ezzati M, et al. Maternal and child undernutrition : global and regional exposures and health consequences. Lancet Ser. 2008;371(9608):243-260. doi: 


\subsection{6/S0140-6736(07)61690-0.}

9. Victora CG, Adair L, Fall C, Hallal PC, Martorell R, Richter L, et al. Materna and Child Undernutrition 2 Maternal and child undernutrition : consequences for adult health and human capital. Lancet Ser 2008;371(9609):340-357. doi: 10.1016/S0140-6736(07)61692-4.

10. Bhutta ZA, Das JK, Rizvi A, Gaff MF, Walker N, Horton S, et al. Evidencebased interventions for improvement of maternal and child nutrition : what can be done and at what cost ? Lancet Ser. 2013;382(9890):452477.

11. Gatahun EA, Abate MG, Abyu DM. Current complementary feeding practice among 6-23 months young children, kemaba woreda south ethiopia. IJFST. 2016;6(1):1-10.

12 Regassa N. Infant and child feeding practices among farming communities in Southern Ethiopia. 2014;16(4):215-222.

13. Haile D, Belachew T, Birhanu G, Setegn T, Biadgilign S. Infant feeding practices among HIV exposed infants using summary index in Sidama Zone, Southern Ethiopia: a cross sectional study. BMC Pediatrics. 2014;14(1):1-9.

14. Demba Gofa District Health sector. Demba Gofa District Health sector : Health profile of 2016. 2016.

15. Demba Gofa Agricuture sector. Demba Gofa District Agriculture sector report of 2016. 2016

16 Golden M, Brennan M, Brennan R, Kaiser R, Seaman J. Measuring Mortality, Nutritional Status, and Food Security in Crisis Situations: SMART METHODOLOGY. 2006;1-129.

17. Arimond M, Ruel MT. Generating Indicators of Appropriate Feeding of Children 6 through 23 months from the KPC 2000+. Washington DC: FANTA / AED; 2003.

18. Ruel MT, Menon P. Creating a Child Feeding Index Using the Demographic and Health Surveys: An Example from Latin America Washington, DC: International Food Policy Research Institute. 2002.

19. Arimond M, Ruel MT. Progress In Developing An Infant And Child Feeding Index: An Example Using The Ethiopia Demographic And Health Survey 2000.Fcnd Discussion Paper No.143. Washington, DC: International Food Policy Research Institute (IFPRI). 2002;143.
20.Tavakol M DR.Making sense of Cronbach 's alpha. Int J Med Ed. 2011;2:53-55. doi: 10.5116/ijme.4dfb.8dfd

21. Tavakol M, Dennick R. Making sense of Cronbach's alpha. 2011;2:5355. doi: $10.5116 / \mathrm{ijme} .4 \mathrm{dfb} .8 \mathrm{dfd}$

22. Garg A, Chadha R. Index for Measuring the Quality of Complementary Feeding Practices in Rural India. 2009;27(6):763-771.

23. Arimond M, Ruel MT. Summary Indicators for Infant and Child Feeding Practices: An Example from the Ethiopia Demographic and Health Survey 2000. Washington, D.C: Food and Nutrition Technical Assistance Project, Academy for Educational Development. 2002.

24. Ruel MT, Menon P. Child Feeding Practices Are Associated with Child Nutritional Status in Latin America: Innovative Uses of the Demographic and Health Surveys. J Nutr. 2002;132(6):1180-1187.

25. Sawadogo PS, Martin-Prevel Y, Savy M, Kameli Y, Traissac P, Traore AS, et al. An Infant and Child Feeding Index Is Associated with the Nutritional Status of 6- to 23-Month-Old Children in Rural Burkina Faso. J Nutr. 2006;136(3):656-663.

26. Srivastava N, Sandhu A. Index for Measuring Child Feeding Practices. Indian J Pediatr. 2007;74(4):363-368.

27.Mukhopadhyay DK, Sinhababu A, Saren AB, Biswas AB. Association of Child Feeding Practices with Nutritional Status of Under-two Slum Dwelling Children: A Community-based Study from West Bengal , India. Indian J Public Heal. 2013;57(3):169-172. doi: 10.4103/0019557X.119819.

28. Arimond M, Ruel MT. Progress In Developing An Infant And Child Feeding Index: An Example Using The Ethiopia Demographic And Health Survey 2000. Washington, DC: International Food Policy Research Institute (IFPRI). 2002.

29. Teshome B, Kogi-makau W, Getahun Z, Taye G. Magnitude and determinants of stunting in children under- five years of age in food surplus region of Ethiopia : The case of West Gojam Zone. Ethiop J Heal Dev. 2009;23(2):98-106. DOI: 10.4314/ejhd.v23i2.53223

30. Fekadu Y, Mesfin A, Haile D, Stoecker BJ. Factors associated with nutritional status of infants and young children in Somali Region, Ethiopia : a cross-sectional study. BMC Public Health. 2015;1-9. DOI: $10.1186 / \mathrm{s} 12889-015-2190-7$ 